\title{
Data analysis at the CMS level-1 trigger: migrating complex selection algorithms from offline analysis and high-level trigger to the trigger electronics
}

\author{
Claudia-Elisabeth Wulz*', Gregor Aradi, Bernhard Arnold, Herbert Bergauer, János \\ Erö, Vasile M. Ghete, Manfred Jeitler, Navid Rad, Johannes Wittmann, Mateusz \\ Zarucki \\ Institute of High Energy Physics, Nikolsdorfergasse 18, A-1050 Vienna, Austria \\ E-mail: Claudia.Wulz@cern.ch, Gregor.Aradi@oeaw.ac.at, \\ Bernhard.Arnold@oeaw.ac.at, Herbert. Bergauer@oeaw.ac.at, \\ Janos.Ero@cern.ch, Vasile.Ghetedoeaw.ac.at, \\ Manfred.Jeitler@cern.ch, Navid.Rad@oeaw.ac.at, \\ Johannes.Wittmann@oeaw.ac.at, Mateusz.Zarucki@oeaw.ac.at
}

\begin{abstract}
With ever increasing luminosity at the LHC, optimum online data selection is becoming more and more important. While in the case of some experiments (LHCb and ALICE) this task is being completely transferred to computer farms, the others - ATLAS and CMS - will not be able to do this in the medium-term future for technological, detector-related reasons. Therefore, these experiments pursue the complementary approach of migrating more and more of the offline and high-level trigger intelligence into the trigger electronics. The presentation illustrates how the level-1 trigger of the CMS experiment and in particular its concluding stage, the so-called "Global Trigger", take up this challenge.
\end{abstract}

The European Physical Society Conference on High Energy Physics

5-12 July 2017

Venice, Italy

* Speaker.

${ }^{\dagger}$ on behalf of the CMS Collaboration. 


\section{Experimental context}

The Large Hadron Collider (LHC) at CERN is currently the most powerful man-made particle accelerator. The CMS experiment [1], one of its multi-purpose detectors, searches for new phenomena beyond the standard model with high priority. The recent performance of the LHC has been particularly impressive [2]. The integrated luminosity for proton-proton collisions at $\sqrt{s}=$ $13 \mathrm{TeV}$ delivered to CMS in 2017 has now exceeded $30 \mathrm{fb}^{-1}$ and is expected to reach $45 \mathrm{fb}^{-1}$ by the end of the year. The associated high instantaneous luminosities (Fig. 1) lead to a large number of simultaneous interactions per beam crossing, yielding about 60 pileup events on average.
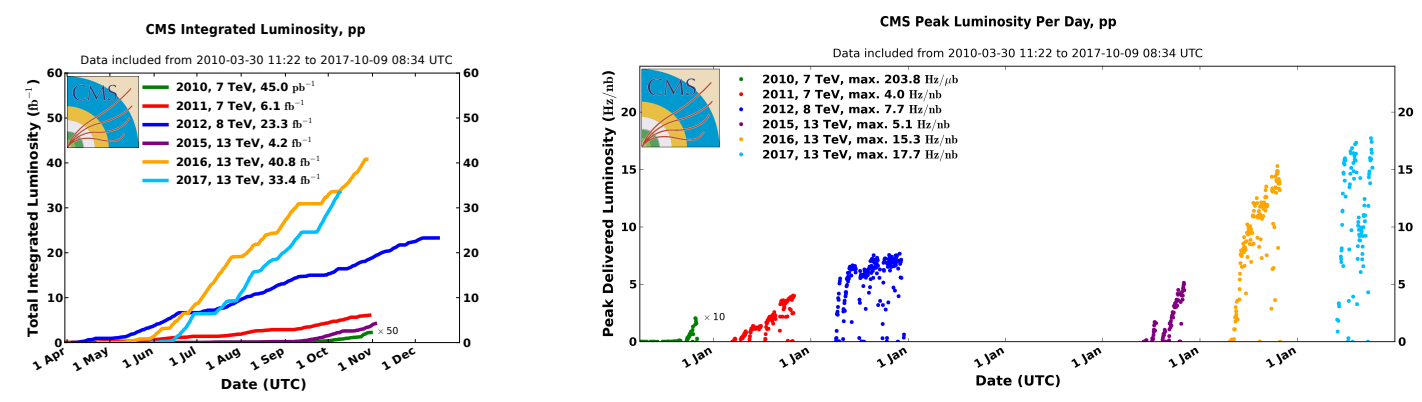

Figure 1: Integrated luminosity delivered to CMS since 2010 (left); CMS peak luminosities per day since 2010 (right) [2].

The future upgraded LHC, the HL-LHC (High-Luminosity LHC), will reach a peak instantaneous luminosity of $7.5 \times 10^{34} \mathrm{~cm}^{-2} \mathrm{~s}^{-1}$ and an average number of up to 200 pileup events. These conditions pose formidable challenges to the experiments, in particular to the on-line trigger and data acquisition systems, which must be able to select and record all collisions interesting for physics, whilst discarding the rest. The CMS trigger [3] is a two-tier system consisting of the level-1 trigger (L1T) and the high-level trigger (HLT). Whilst the L1T is made of fast, customised electronics, the HLT is implemented in a farm of off-the-shelf processors. The final decision stage of the L1T is the Global Trigger.

\section{Global Trigger}

The Global Trigger [4] represents the highest stage of the L1 hierarchy [5], as shown in Fig. 2. It receives and synchronises inputs from the calorimeters, the muon system as well as from beam radiation and luminosity detectors. It issues the L1 trigger decision based on the results of over five hundred logic algorithms running in parallel. It is possible to prescale algorithms, depending on luminosity. The set of algorithms represents the trigger menu. The Global Trigger of the so-called phase-I upgrade [6, 7] is implemented in $\mu$ TCA technology. Processing is performed with large field programmable gate arrays from the XILINX Virtex-7 family on each of the six MP7 modules [8]. Its current maximum output rate to the HLT is $100 \mathrm{kHz}$, whilst for the HL-LHC $1 \mathrm{MHz}$ is foreseen. 

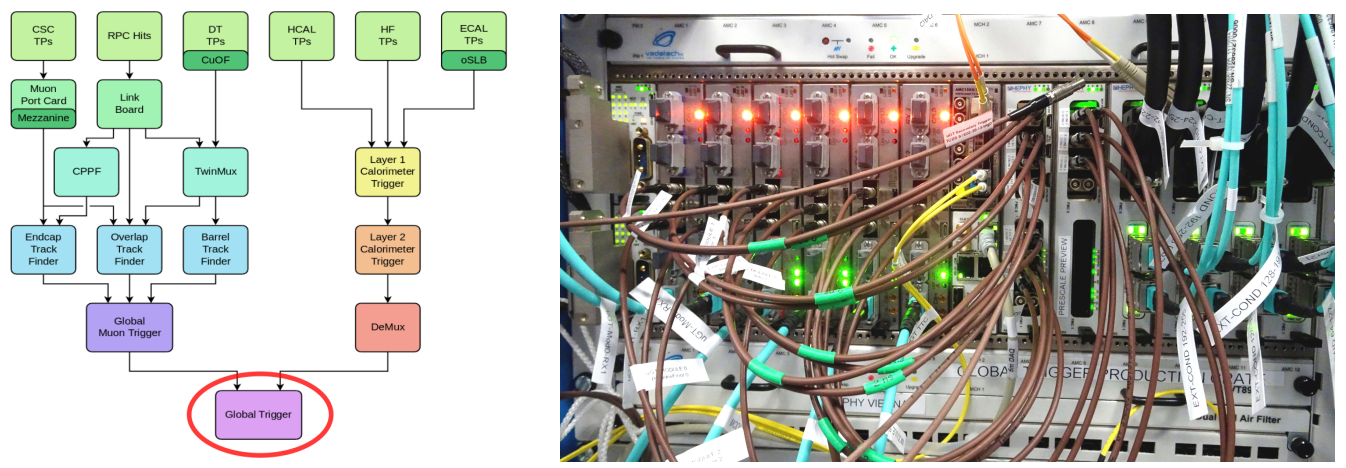

Figure 2: The CMS level-1 trigger (left) [5]; the Global Trigger crate at the experimental site (right).

\section{Global Trigger Algorithms}

Algorithms are based on candidate trigger objects such as electrons/photons, taus, jets, muons, transverse energy sums, or missing transverse momentum. Objects contain information on energy or momentum, location and quality, therefore complex selection criteria can be applied, in addition to the standard ones relying on energy or momentum thresholds. Combinations of different object types called cross-triggers are possible. Topological conditions may be applied, and complex, analysis-like variables such as invariant mass can be used for triggering. In order to save processing resources, the following approximation is used to calculate the invariant mass $M_{\text {inv }}$ of two objects with transverse momenta $p_{\mathrm{T} 1}, p_{\mathrm{T} 2}$, pseudorapidities $\eta_{1}, \eta_{2}$ and azimuthal angles $\varphi_{1}, \varphi_{2}$ :

$$
M_{\mathrm{inv}} \approx \sqrt{2 p_{T 1} p_{T 2}\left[\cosh \left(\eta_{1}-\eta_{2}\right)-\cos \left(\varphi_{1}-\varphi_{2}\right)\right]}
$$

It is useful for example in selecting vector boson fusion processes, where two high-energy jets $\left(j_{f}\right)$ are present in the forward regions, as shown in Fig. 3 (left). Fig. 3 (right) shows an on-line
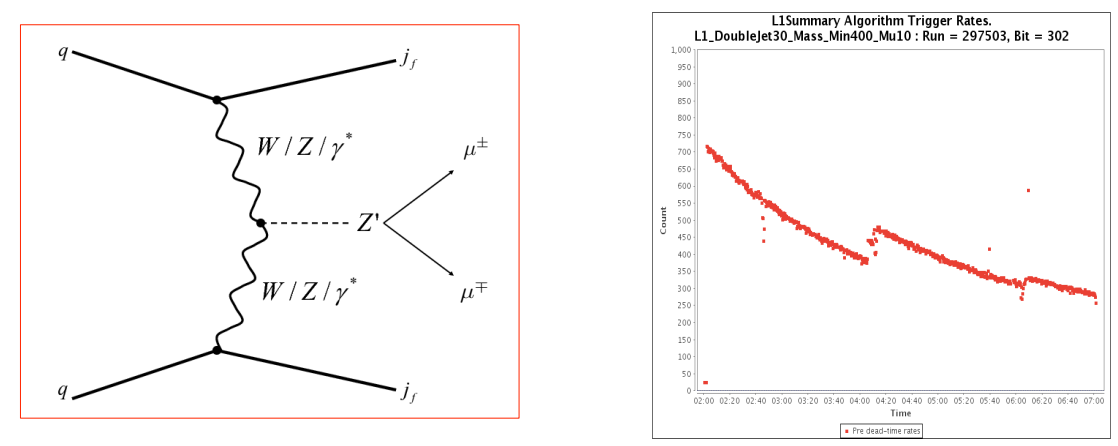

Figure 3: Example of a vector boson fusion process (left); level-1 trigger rates for a vector boson fusion process (right).

histogram with level-1 trigger count rates for an algorithm that requires two jets with transverse momenta above $30 \mathrm{GeV}$ and an invariant mass larger than $400 \mathrm{GeV}$, and a muon with $p_{\mathrm{T}}>10 \mathrm{GeV}$, as a function of the time elapsed during a specific LHC data taking period. 


\section{Trigger Menu Editor}

There is very high flexibility in defining trigger menus. For each menu, the maximal number of algorithms is 512, which represents an increase of a factor of four compared to the previous system. A tool for physicists to design the menu algorithms, in particular complex ones, has been developed [9]. An example screenshot of the user interface of the Trigger Menu Editor is depicted in Fig. 4. The trigger menu is stored as an XML file, and a VHDL producer translates it into code that can be synthesised.

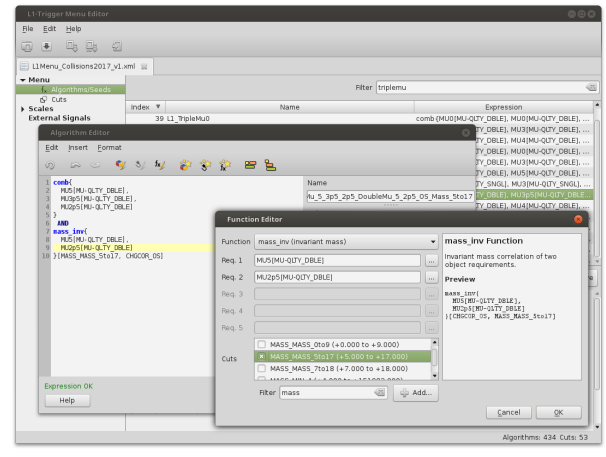

Figure 4: The Trigger Menu Editor in operation.

\section{Conclusions}

The CMS Global Trigger, the final stage of the hardware-based level-1 trigger, offers the possibility to implement analysis-like algorithms during the on-line event selection process. This feature is of particular importance in view of challenging LHC luminosity and pileup conditions. Raising energy or momentum thresholds, which could have a negative impact on the physics potential of the experiment, can thus be avoided, and highly selective physics data can be recorded.

\section{References}

[1] CMS Collaboration, JINST 3 (2008) S08004. doi:10.1088/1748-0221/3/08/S08004

[2] CMS Collaboration, https://twiki.cern.ch/twiki/bin/view/CMSPublic/LumiPublicResults

[3] CMS Collaboration, http://iopscience.iop.org/article/10.1088/1748-0221/12/01/P01020 Journal of Instrumentation Vol 12, No 1, p P01020 (2017)

[4] J. Wittmann et al., Journal of Instrumentation Vol 12, No 1, p C01046 (2017)

[5] Th. Reis, https://treis.web.cern.ch/treis/cms/11_muon_upgrade/docs/ (2017)

[6] A. Tapper and D. Acosta (editors), CERN-LHCC-2013-011. CMS-TDR-12 (2013)

[7] http://globaltrigger.hephy.at, Institute of High Energy Physics, Vienna, Austria.

[8] A. Rose et al., Journal of Instrumentation Vol 7, No 12, p C12024 (2012)

[9] T. Matsushita and B. Arnold, Journal of Physics: Conference Series Vol 664, No 8, p 082031 (2015) 